\title{
Hearing assessment after meningitis and meningococcal disease
}

\author{
Andrew Riordan, Alistair Thomson, Judith Hodgson
}

\begin{abstract}
A method to increase audiology referral after meningitis or meningococcal disease was audited in 89 children. A standardised proforma increased referrals from $78 \%$ to $96 \%$ over a two year period. However, only $73 \%$ of children had a hearing test. The major reason for hearing not being tested changed from non-referral to nonattendance.

(Arch Dis Child 1995; 72: 441-442)
\end{abstract}

Keywords: meningitis, sensorineural deafness, audit.

Sensorineural hearing loss occurs in $10 \%$ of children surviving bacterial meningitis or meningococcal disease. ${ }^{12}$ Partial or unilateral hearing loss can cause linguistic disabilities, but may be missed by informal testing at the bedside or in outpatients. All children should therefore have a formal audiological assessment after meningitis. ${ }^{2}$ Hearing loss can also occur after meningococcal septicaemia without meningitis. ${ }^{3}$ All children with meningococcal disease, not just those with meningitis, should therefore have audiological follow up.

A recent audit in our hospital found that only $75 \%$ of survivors of meningitis had a hearing assessment. ${ }^{4}$ The major reason for hearing not being assessed was non-referral. As a result of our audit a simpler method of referral was adopted.

We now present an audit of this new referral method. The standard set was for all children with meningococcal disease or bacterial meningitis to be referred for and have a formal audiological assessment.

\section{Methods}

From 1 April 1993 a standardised proforma for audiology referral was available on all medical wards. Before this referral had been by letter. Medical staff were asked to complete the form when discharging children with meningitis or meningococcal disease. Forms were sent to one of us $(\mathrm{JH})$ at community services, and forwarded to the appropriate local audiology service.

Audiology referrals and hearing test results after meningitis and meningococcal disease. Data shown as number (\%)

Health

A Thomson

Community Child

Health

J Hodgson

Correspondence to:

Dr A Riordan, 20 Rosefield

Avenue, Bebington, Wirral

L63 5JT.

Accepted 27 January 1995
Referred by hospita

Other referral

Did not attend hearing test

Hearing tested

Sensorineural loss

\begin{tabular}{cc}
$30(73)$ & $42(88)$ \\
$2(5)$ & $4(8)$ \\
$32(78)$ & $46(96)$ \\
$5(12)$ & $11(23)$ \\
$27(66)$ & $35(73)$ \\
$2(5)$ & $3(6)$ \\
\hline
\end{tabular}

A list of children with a discharge diagnosis of bacterial meningitis or meningococcal disease was compiled for the year before, and the year after the introduction of the new referral method as part of a prospective study (A Riordan, unpublished data). After one year of the new referral method, audiology records were studied to see how many children had been referred and how many had had a hearing test. The referral rates were compared with those for the previous year.

\section{Results}

During the two years audited 96 children were directly admitted to our hospital with bacterial meningitis or meningococcal disease. Eighty nine children survived and were included in our audit. Referrals for audiology were only made for these children, except for one other child. This child had a possible viral meningitis, and was excluded from the audit.

The number of children admitted was similar for the two 12 month periods (table). The causative organisms were also similar (data not shown). However, the proportion of children known to the audiology services was much greater during the latter year $(78 \% v 96 \%)$ (table). This was in part due to an increase in referrals on the new referral form, but also due to increased referrals from other sources. Despite the increased referrals for audiology, the proportion of children who had their hearing tested increased to only $73 \%$. This was due to an increase in non-attendance for hearing test after referral. Five of the $62(8 \%)$ children assessed were found to have sensorineural hearing loss.

\section{Discussion}

Having identified non-referral as the major reason for hearing not being assessed after meningitis, ${ }^{4}$ we implemented a new referral method. This audit shows the effectiveness of this simpler method of referral, with $96 \%$ of cases now being known to the audiology services. This improvement was due to an increasing referral rate from medical staff $(73 \%$ to $88 \%$ ) as well as from other professionals. The proportion of children who actually had a hearing assessment changed very little, however $(66 \%$ to $73 \%)$, and is similar to that previously reported by ourselves ${ }^{4}$ and others. ${ }^{15}$ The main reason for non-assessment changed during the audit from failure to refer to failure to attend for audiology.

The increasing rate for those who did not attend is worrying. A non-attended appointment at a specialist centre wastes resources, 
and is more difficult to rectify than nonreferral. Hospital staff can be made aware of the need for hearing assessment after meningitis in large groups. Parents, however, will need to be seen individually and may not appreciate the need to return for hearing assessment.

Eleven of the 16 children who defaulted audiology appointments did attend paediatric outpatients. Encouragement to attend hearing assessment then, or appointments on the same day, could help increase the number of children tested. Such a system will require close cooperation between audiology and paediatric services and continuous audit.

Increasing referral to the audiology services after meningitis and meningococcal disease up to $96 \%$ did not substantially increase the number of children assessed. Strategies to decrease non-attendance now need to be devised and the audit repeated.

We would like to thank Frank McIntyre for help in data collection and the Johanne Holly Trust for financial support.

1 Dawson JA, Wardle R. Detection and prevalence of hearing loss in a cohort of children following serogroup $B$ loss in a cohort of children following serogroup B 104: 99-102.

2 Fortnum HM. Hearing impairment after bacterial meningitis: a review. Arch Dis Child 1992; 67: 1128-33.

3 Thomson A, Marzouk O. Endotoxin induced cochlear damage. Arch Dis Child 1991; 66: 907-8.

4 Riordan A, Thomson A, Hodgson J, Hart A. Children who are seen but not referred: hearing assessment after bacterial meningitis. Br f Audiol 1993; 27: 375-7.

5 Fortnum HM, Davis AC. Hearing impairment in children after bacterial meningitis: incidence and resource implications. Br F Audiol 1993; 27: 43-52. 